\title{
Anderson localization for the almost Mathieu operator in the exponential regime
}

\author{
Wencai Liu ${ }^{1}$ and Xiaoping Yuan ${ }^{1}$
}

Abstract. For the almost Mathieu operator

$$
\left(H_{\lambda, \alpha, \theta} u\right)_{n}=u_{n+1}+u_{n-1}+2 \lambda \cos 2 \pi(\theta+n \alpha) u_{n},
$$

Jitomirskaya conjectures that for a.e. $\theta, H_{\lambda, \alpha, \theta}$ satisfies Anderson localization if $|\lambda|>e^{\beta}$. Avila and Jitomirskaya verify this for $|\lambda|>e^{\frac{16}{9} \beta}$. In the present paper, we extend their result to regime $|\lambda|>e^{\frac{3}{2} \beta}$.

Mathematics Subject Classification (2010). 34K08, 47A10, 47A35.

Keywords. Anderson localization, almost Mathieu operator.

\section{Introduction}

The almost Mathieu operator (AMO) is the (discrete) quasi-periodic Schrödinger operator on $\ell^{2}(\mathbb{Z})$ :

$$
\left(H_{\lambda, \alpha, \theta} u\right)_{n}=u_{n+1}+u_{n-1}+\lambda v(\theta+n \alpha) u_{n}, \quad \text { with } v(\theta)=2 \cos 2 \pi \theta,
$$

where $\lambda$ is the coupling, $\alpha$ is the frequency, and $\theta$ is the phase.

$H_{\lambda, \alpha, \theta}$ is a tight binding model for the Hamiltonian of an electron in a onedimensional lattice or in a two-dimensional lattice, subject to a perpendicular (uniform) magnetic field (through a Landau gauge) [9]. For more applications in physics, we refer the reader to [13] and the references therein.

Besides its relations to some fundamental problems in physics, the AMO itself is also fascinating because of its remarkable richness of the related spectral theory. In B. Simon's list of Schrödinger operator problems for the twenty-first century [14] , there are three problems about the AMO. The spectral theory of 
AMO has attracted many authors, for example, Avila and Jitomirskaya[1] and [2], Avila and Krikorian [3], Bourgain [5] and [6], Jitomirskaya and Simon [12], and so on.

Anderson localization (i.e., only pure point spectrum with exponentially decaying eigenfunctions) is not only meaningful in physics, but also relates to some problems of the quasi-periodic Schrödinger operator, such as the reducibility of cocycles via Aubry duality [8] and the ten Martini problem (Cantor spectrum conjecture) [1].

For $\alpha \in \mathbb{Q}$, it is easy to verify that $H_{\lambda, \alpha, \theta}$ has no eigenvalues, let alone Anderson localization. Thus, in the present paper, we always assume $\alpha \in \mathbb{R} \backslash \mathbb{Q}$.

For simplicity, we say $H_{\lambda, \alpha, \theta}$ satisfies $A L$ if for a.e. phase $\theta, H_{\lambda, \alpha, \theta}$ satisfies Anderson localization.

Jitomirskaya [10] conjectures ${ }^{1}$ that $H_{\lambda, \alpha, \theta}$ satisfies AL for $|\lambda|>e^{\beta}$, where

$$
\beta=\beta(\alpha)=\limsup _{n \rightarrow \infty} \frac{\ln q_{n+1}}{q_{n}},
$$

and $\frac{p_{n}}{q_{n}}$ is the continued fraction approximants to $\alpha$. One usually calls set

$$
\{\alpha \in \mathbb{R} \backslash \mathbb{Q}: \beta(\alpha)>0\}
$$

exponential regime and set

$$
\{\alpha \in \mathbb{R} \backslash \mathbb{Q}: \beta(\alpha)=0\}
$$

sub-exponential regime.

This conjecture is optimal in some way. On the one hand, for every $\alpha$ there is a generic set of $\theta$ for which there is no eigenvalues [12]. On the other hand, if $|\lambda| \leq e^{\beta}$, for every $\theta, H_{\lambda, \alpha, \theta}$ is expected to have no localized eigenfunctions (i.e., exponentially decaying eigenfunctions), see footnote 3 in [1].

In [11], Jitomirskaya proves that $H_{\lambda, \alpha, \theta}$ satisfies AL if $\alpha \in D C$ and $|\lambda|>1$. In fact Jitomirskaya's arguments also hold for $\beta(\alpha)=0$ and $|\lambda|>1$. In order to prove the ten Martini problem, Avila and Jitomirskaya [1] show that $H_{\lambda, \alpha, \theta}$ satisfies AL if $|\lambda|>e^{\frac{16}{9} \beta}$. You and Zhou [15] prove that for almost every phase $\theta$, the eigenvalues of operator $H_{\lambda, \alpha, \theta}$ with exponentially decaying eigenfunctions are dense in the spectrum if $|\lambda|>C e^{\beta}$, where $C$ is a large absolute constant. We also should point out that they did not show the Anderson Localization. In the present paper, we verify the conjecture in regime $|\lambda|>e^{\frac{3}{2} \beta}$, i.e., the following theorem.

\footnotetext{
${ }^{1}$ After submitting the present paper, we learned of that Avila, You, and Zhou claimed they completed the conjecture. (Their preprint is not available yet.)
} 
Theorem 1.1 (main theorem). Let $\alpha \in \mathbb{R} \backslash \mathbb{Q}$ be such that $\beta=\beta(\alpha)<\infty$, then for almost every phase $\theta, H_{\lambda, \alpha, \theta}$ satisfies Anderson localization if $|\lambda|>e^{\frac{3}{2} \beta}$.

We investigate AL following the general scheme of Avila and Jitomirskaya, but some estimates are more subtle.

The present paper is organized as follows. In $\S 2$, we give some preliminary notions and facts which are taken from other authors, such as Avila and Jitomirskaya [1], Bourgain [6], and so on. In $\S 3$, we set up the regularity of resonant $y$ if $|\lambda|>e^{\frac{3}{2} \beta}$. In $\S 4$, we give the proof of main theorem by block resolvent expansion.

\section{Preliminaries and some known results}

It is well known that Anderson localization for a self-adjoint operator $H$ on $\ell^{2}$ is equivalent to the following statements [4].

Assume $\phi$ is an extended state, i.e.,

$$
H \phi=E \phi \quad \text { with } E \in \Sigma(H) \text { and }|\phi(k)| \leq(1+|k|)^{C},
$$

where $\Sigma(H)$ is the spectrum of self-adjoint operator $H$. Then there exists some constant $c>0$ such that

$$
|\phi(k)|<e^{-c|k|} \text { for } k \rightarrow \infty .
$$

We will actually prove a slightly more precise version of Theorem 1.1. Let

$$
\mathcal{R}_{1}=\left\{\theta:|\sin \pi(2 \theta+k \alpha)| \leq k^{-2} \text { holds for infinitely many } k, k \in \mathbb{Z}\right\},
$$

and

$$
\mathcal{R}_{2}=\{\theta: \text { there exists } s \in \mathbb{Z} \text { such that } 2 \theta+s \alpha \in \mathbb{Z}\} .
$$

Clearly,

$$
\mathcal{R}=\mathcal{R}_{1} \cup \mathcal{R}_{2}
$$

has zero Lebesgue measure.

Theorem 2.1. Let $\alpha \in \mathbb{R} \backslash \mathbb{Q}$ be such that $\beta=\beta(\alpha)<\infty$, then $H_{\lambda, \alpha, \theta}$ satisfies Anderson localization if $\theta \notin \mathcal{R}$ and $|\lambda|>e^{\frac{3}{2} \beta}$. 
If $\alpha$ satisfies $\beta(\alpha)=0$, Theorem 2.1 has been proved by Jitomirskaya [10]. Thus in the present paper, we fix $\alpha \in \mathbb{R} \backslash \mathbb{Q}$ such that $0<\beta(\alpha)<\infty$. Unless stated otherwise, we always assume $\lambda>e^{\frac{3}{2} \beta}$ (for $\lambda<-e^{\frac{3}{2} \beta}$, note that $H_{\lambda, \alpha, \theta}=$ $H_{-\lambda, \alpha, \theta+\frac{1}{2}}$ ), $\theta \notin \mathcal{R}$ and $E \in \Sigma_{\lambda, \alpha}$ (denote by $\Sigma_{\lambda, \alpha}$ the spectrum of operator $H_{\lambda, \alpha, \theta}$ since the spectrum does not depend on $\theta$ ). Since this does not change any of the statements, sometimes the dependence of parameters $E, \lambda, \alpha, \theta$ will be ignored in the following.

Given an extended state $\phi$ of $H_{\lambda, \alpha, \theta}$, without loss of generality one can assume $\phi(0)=1$. Our objective is to prove that there exists some $c>0$ such that

$$
|\phi(k)|<e^{-c|k|} \text { for } k \rightarrow \infty .
$$

Let us denote

$$
P_{k}(\theta)=\operatorname{det}\left(R_{[0, k-1]}\left(H_{\lambda, \alpha, \theta}-E\right) R_{[0, k-1]}\right) .
$$

It is easy to see that $P_{k}(\theta)$ is an even function of $\theta+\frac{1}{2}(k-1) \alpha$ and can be written as a polynomial of degree $k$ in $\cos 2 \pi\left(\theta+\frac{1}{2}(k-1) \alpha\right)$ :

$$
P_{k}(\theta)=\sum_{j=0}^{k} c_{j} \cos ^{j} 2 \pi\left(\theta+\frac{1}{2}(k-1) \alpha\right) \triangleq Q_{k}\left(\cos 2 \pi\left(\theta+\frac{1}{2}(k-1) \alpha\right)\right) .
$$

Let

$$
A_{k, r}=\left\{\theta \in \mathbb{R}:\left|Q_{k}(\cos 2 \pi \theta)\right| \leq e^{(k+1) r}\right\}
$$

with $k \in \mathbb{N}$ and $r>0$.

Lemma 2.1 ([1], p.16). The following inequality holds

$$
\lim _{k \rightarrow \infty} \sup _{\theta \in \mathbb{R}} \frac{1}{k} \ln \left|P_{k}(\theta)\right| \leq \ln \lambda .
$$

By Cramer's rule (see [6], p. 15, for example) for given $x_{1}$ and $x_{2}=x_{1}+k-1$, with $y \in I=\left[x_{1}, x_{2}\right] \subset \mathbb{Z}$, one has

$$
\left|G_{I}\left(x_{1}, y\right)\right|=\left|\frac{P_{x_{2}-y}(\theta+(y+1) \alpha)}{P_{k}\left(\theta+x_{1} \alpha\right)}\right|,
$$

and

$$
\left|G_{I}\left(y, x_{2}\right)\right|=\left|\frac{P_{y-x_{1}}\left(\theta+x_{1} \alpha\right)}{P_{k}\left(\theta+x_{1} \alpha\right)}\right| .
$$

By Lemma 2.1, the numerators in (2.1) and (2.2) can be bounded uniformly with respect to $\theta$. Namely, for any $\varepsilon>0$,

$$
\left|P_{n}(\theta)\right| \leq e^{(\ln \lambda+\varepsilon) n}
$$

for $n$ large enough. 
Definition 2.1. Fix $t>0,0<\delta<1 / 2$. A point $y \in \mathbb{Z}$ will be called $(t, k)$-regular with $\delta$ if there exists an interval $\left[x_{1}, x_{2}\right]$ containing $y$, where

$$
x_{2}=x_{1}+k-1 \text {, }
$$

such that

$$
\left|G_{\left[x_{1}, x_{2}\right]}\left(y, x_{i}\right)\right|<e^{-t\left|y-x_{i}\right|} \text { and }\left|y-x_{i}\right| \geq \delta k, \quad \text { for } i=1,2 .
$$

It is easy to check ([6], p. 61) that

$$
\phi(x)=-G_{\left[x_{1}, x_{2}\right]}\left(x_{1}, x\right) \phi\left(x_{1}-1\right)-G_{\left[x_{1}, x_{2}\right]}\left(x, x_{2}\right) \phi\left(x_{2}+1\right),
$$

where $x \in I=\left[x_{1}, x_{2}\right] \subset \mathbb{Z}$. Our strategy is to establish the $(t, k(y))$-regularity for every large $y$, then localized property is easy to obtain by (2.4) and the block resolvent expansion.

Definition 2.2. We say that the set $\left\{\theta_{1}, \ldots, \theta_{k+1}\right\}$ is $\epsilon$-uniform if

$$
\max _{x \in[-1,1]} \max _{i=1, \ldots, k+1} \prod_{j=1, j \neq i}^{k+1} \frac{\left|x-\cos 2 \pi \theta_{j}\right|}{\left|\cos 2 \pi \theta_{i}-\cos 2 \pi \theta_{j}\right|}<e^{k \epsilon} .
$$

Lemma 2.2 ([1], Lemma 9.3). Suppose that $\left\{\theta_{1}, \ldots, \theta_{k+1}\right\}$ is $\epsilon_{1}$-uniform. Then there exists some $\theta_{i}$ in set $\left\{\theta_{1}, \ldots, \theta_{k+1}\right\}$ such that $\theta_{i} \notin A_{k, \ln \lambda-\epsilon}$ if $\epsilon>\epsilon_{1}$ and $k$ is sufficiently large.

Assume without loss of generality that $y>0$. Fix a sufficient small constant $\eta$ (that will be determined in Theorem 3.3). Define

$$
b_{n}=\max \left\{\eta q_{n-1}, q_{n}^{8 / 9}\right\},
$$

where $q_{n}$ is given by (1.2), and find $n$ such that

$$
b_{n} \leq y<b_{n+1} .
$$

We will distinguish two cases:

(i) $\left|y-\ell q_{n}\right| \leq b_{n}$ for some $\ell \geq 1$, called resonance;

(ii) $\left|y-\ell q_{n}\right|>b_{n}$ for all $\ell \geq 0$, called non-resonance.

For the non-resonant $y$, Avila and Jitomirskaya have established the regularity for $y$. We give the theorem directly. 
Theorem 2.2 ([1], Lemma 9.4). Assume $\theta \notin \mathcal{R}, \lambda>e^{\beta}$, and that $y$ is nonresonant. Let $s \in \mathbb{N}$ be the largest number such that

$$
s q_{n-1} \leq \operatorname{dist}\left(y,\left\{\ell q_{n}\right\}_{\ell \geq 0}\right) \text {. }
$$

Then for all $\varepsilon>0$ and $n$ large enough, the following hold:

(i) if $s \geq 1$, then $y$ is $\left(\ln \lambda+9 \ln \left(s q_{n-1} / q_{n}\right) / q_{n-1}-\varepsilon, 2 s q_{n-1}-1\right)$-regular with

$$
\delta=\frac{1}{8}
$$

(ii) If $s=0$, then $y$ is either $\left(\ln \lambda-\varepsilon, 2\left[q_{n-1} / 2\right]-1\right)-$, or $\left(\ln \lambda-\varepsilon, 2\left[q_{n} / 2\right]-1\right)-$, or $\left(\ln \lambda-\varepsilon, 2 q_{n-1}-1\right)$-regular with

$$
\delta=\frac{\eta}{2}
$$

Remark 2.1. Avila and Jitomirskaya let

$$
b_{n}=\max \left\{\frac{q_{n-1}}{20}, q_{n}^{\frac{8}{9}}\right\}
$$

(i.e., $\eta=\frac{1}{20}$ )in defining resonance and nonresonance, and they obtain $y$ is regular with $\delta=\frac{1}{8}$ in case (i), and with $\delta=\frac{1}{40}$ (i.e., $\delta=\frac{\eta}{2}$ ) in case (ii). We give the general definition of resonance and nonresonance, and Theorem 2.2 also holds. The analysis follows from Avila-Jitomirskaya's arguments, we omit the proof.

Lemma 2.3 ([1], Lemma 9.8). Let $m \in \mathbb{N}$ be such that

$$
m<\frac{q_{r+1}}{10 q_{n}}
$$

where $r \geq n$. Given a integer sequence $\left|m_{k}\right| \leq m-1, k=1, \ldots, q_{n}$, let $1 \leq k_{0} \leq$ $q_{n}$ be such that

$$
\left|\sin \pi\left(x+\left(k_{0}+m_{k_{0}} q_{r}\right) \alpha\right)\right|=\min _{1 \leq k \leq q_{n}}\left|\sin \pi\left(x+\left(k+m_{k} q_{r}\right) \alpha\right)\right|,
$$

then

$$
\begin{gathered}
\left|\sum_{\substack{k=1 \\
k \neq k_{0}}}^{q_{n}} \ln \right| \sin \pi\left(x+\left(k+m_{k} q_{r}\right) \alpha\right)\left|+\left(q_{n}-1\right) \ln 2\right| \\
<C \ln q_{n}+C\left(\Delta_{n}+(m-1) \Delta_{r}\right) q_{n} \ln q_{n},
\end{gathered}
$$

where $\Delta_{n}=\left|q_{n} \alpha-p_{n}\right|$. 


\section{Regularity for resonant $y$}

In this section, we mainly concern the regularity for resonant $y$. In this condition $y>\frac{q_{n}}{2}$. Thus by the definition of resonance, there exists some positive integer $\ell$ with $1 \leq \ell \leq q_{n+1}^{8 / 9} / q_{n}$ such that $\left|y-\ell q_{n}\right| \leq b_{n}$. Fix the positive integer $\ell$ and set $I_{1}, I_{2} \subset \mathbb{Z}$ as

$$
I_{1}=\left[-\left[\frac{2}{3} q_{n}\right],\left[\frac{2}{3} q_{n}\right]-2\right]
$$

and

$$
I_{2}=\left[(\ell-1) q_{n}+\left[\frac{2}{3} q_{n}\right]-1,(\ell+1) q_{n}-\left[\frac{2}{3} q_{n}\right]-1\right]
$$

and let

$$
\theta_{j}=\theta+j \alpha \quad \text { for } j \in I_{1} \cup I_{2} .
$$

The set $\left\{\theta_{j}\right\}_{j \in I_{1} \cup I_{2}}$ consists of $2 q_{n}$ elements.

Note that, below, we replace $I=\left[x_{1}, x_{2}\right] \cap \mathbb{Z}$ with $I=\left[x_{1}, x_{2}\right]$ for simplicity, and assume $\varepsilon>0$ is sufficiently small.

We will use the following three steps to establish regularity for $y$.

S TEP 1 . For any $\varepsilon>0$, we set up the $\frac{\beta}{2}+\varepsilon$-uniformity of $\left\{\theta_{j}\right\}$ where $\theta_{j}=\theta+j \alpha$ and $j$ ranges through $I_{1} \cup I_{2}$. By Lemma 2.2, there exists some $j_{0}$ with $j_{0} \in I_{1} \cup I_{2}$ such that $\theta_{j_{0}} \notin A_{2 q_{n}-1, \ln \lambda-\frac{\beta}{2}-C \eta}$.

Step 2. We show that for all $j \in I_{1}, \theta_{j} \in A_{2 q_{n}-1, \ln \lambda-\frac{\beta}{2}-C \eta}$ if $\lambda>e^{\frac{3}{2} \beta}$. Thus there exists $\theta_{j_{0}} \notin A_{2 q_{n}-1, \ln \lambda-\frac{\beta}{2}-C \eta}$ for some $j_{0} \in I_{2}$.

Step 3. We establish the regularity for $y$.

Remark 3.1. In [1], Avila and Jitomirskaya construct

$$
\begin{aligned}
& I_{1}=\left[-\left[\frac{5}{8} q_{n}\right],\left[\frac{5}{8} q_{n}\right]-1\right], \\
& I_{2}=\left[(\ell-1) q_{n}+\left[\frac{5}{8} q_{n}\right],(\ell+1) q_{n}-\left[\frac{5}{8} q_{n}\right]-1\right],
\end{aligned}
$$

and set

$$
\theta_{j}=\theta+j \alpha \quad \text { for } j \in I_{1} \cup I_{2} \text {. }
$$


They use the above three steps to establish the regularity of $y$. More precisely, firstly, they establish the $\left(\frac{\beta}{2}+\varepsilon\right)$-uniformity of $\left\{\theta_{j}\right\}$ and that there exists $\theta_{j_{0}} \notin$ $A_{2 q_{n}-1, \ln \lambda-\frac{\beta}{2}-2 \varepsilon}$ for some $j_{0} \in I_{1} \cup I_{2}$. Secondly, they prove that for all $j \in I_{1}$, $\theta_{j} \in A_{2 q_{n}-1, \ln \lambda-\frac{\beta}{2}-2 \varepsilon}$, and thus there exists $\theta_{j_{0}} \notin A_{2 q_{n}-1, \ln \lambda-\frac{\beta}{2}-2 \varepsilon}$ for some $j_{0} \in I_{2}$, if $\lambda>e^{\frac{16}{9} \beta}$. Thirdly, they set up the regularity of $y$. In the present paper, we reconstruct $I_{1}$ and $I_{2}$, and show that the three steps also hold.

Recall that

$$
\|k \alpha\|_{\mathbb{R} / \mathbb{Z}} \geq \Delta_{n}, \quad \text { for all } 1 \leq k<q_{n+1},
$$

and

$$
\frac{1}{2 q_{n+1}} \leq \Delta_{n} \leq \frac{1}{q_{n+1}}
$$

where

$$
\|x\|_{\mathbb{R} / \mathbb{Z}}=\min _{j \in \mathbb{Z}}|x-j| .
$$

STEP 1 . We establish the $\left(\frac{\beta}{2}+\varepsilon\right)$-uniformity for $\left\{\theta_{j}\right\}_{j \in I_{1} \cup I_{2}}$.

In Lemma 2.3, let $r=n$ and $m=\ell \leq q_{n+1}^{8 / 9} / q_{n}$, one has

$$
\left(\Delta_{n}+(m-1) \Delta_{r}\right) q_{n}=\ell \Delta_{n} q_{n} \leq C,
$$

since $\Delta_{n} \leq \frac{1}{q_{n+1}}$ by (3.2). Moreover, we obtain the following lemma.

Lemma 3.1. Given a integer sequence $\left|m_{k}\right| \leq \ell-1, k=1, \ldots, q_{n}$, let $1 \leq k_{0} \leq q_{n}$ be such that

$$
\left|\sin \pi\left(x+\left(k_{0}+m_{k_{0}} q_{n}\right) \alpha\right)\right|=\min _{1 \leq k \leq q_{n}}\left|\sin \pi\left(x+\left(k+m_{k} q_{n}\right) \alpha\right)\right| .
$$

Then

$$
\begin{aligned}
-\left(q_{n}-1\right) \ln 2-C \ln q_{n} & \leq \sum_{\substack{k=1 \\
k \neq k_{0}}}^{q_{n}} \ln \left|\sin \pi\left(x+\left(k+m_{k} q_{n}\right) \alpha\right)\right| \\
& \leq-\left(q_{n}-1\right) \ln 2+C \ln q_{n} .
\end{aligned}
$$


Theorem 3.1. For all $\varepsilon>0$, the set $\left\{\theta_{j}\right\}_{j \in I_{1} \cup I_{2}}$ is $\left(\frac{\beta}{2}+\varepsilon\right)$-uniform for $\theta \notin \mathcal{R}$ and sufficiently large $n$.

Proof. Let

$$
I_{1}^{\prime}=\left[-\left[\frac{2}{3} q_{n}\right],-\left[\frac{2}{3} q_{n}\right]+q_{n}-1\right]
$$

and

$$
I_{2}^{\prime}=\left[-\left[\frac{2}{3} q_{n}\right]+q_{n},\left[\frac{2}{3} q_{n}\right]-2\right] \cup\left[(\ell-1) q_{n}+\left[\frac{2}{3} q_{n}\right]-1,(\ell+1) q_{n}-\left[\frac{2}{3} q_{n}\right]-1\right] .
$$

Clearly, both $\left\{\theta_{j}\right\}_{j \in I_{1}^{\prime}}$ and $\left\{\theta_{j}\right\}_{j \in I_{2}^{\prime}}$ consist of $q_{n}$ elements, and $I_{1}^{\prime} \cup I_{2}^{\prime}=I_{1} \cup I_{2}$. In (2.5), let $x=\cos 2 \pi a, k=2 q_{n}-1$ and take the logarithm. Thus in order to prove the theorem, it suffices to show that for any $a \in \mathbb{R}$ and $i \in I_{1}^{\prime} \cup I_{2}^{\prime}$,

$$
\begin{aligned}
\ln & \prod_{j \in I_{1}^{\prime} \cup I_{2}^{\prime}, j \neq i} \frac{\left|\cos 2 \pi a-\cos 2 \pi \theta_{j}\right|}{\left|\cos 2 \pi \theta_{i}-\cos 2 \pi \theta_{j}\right|} \\
= & \sum_{j \in I_{1}^{\prime} \cup I_{2}^{\prime}, j \neq i} \ln \left|\cos 2 \pi a-\cos 2 \pi \theta_{j}\right|-\sum_{j \in I_{1}^{\prime} \cup I_{2}^{\prime}, j \neq i} \ln \left|\cos 2 \pi \theta_{i}-\cos 2 \pi \theta_{j}\right| \\
< & \left(2 q_{n}-1\right)\left(\frac{\beta}{2}+\varepsilon\right) .
\end{aligned}
$$

Without loss of generality assume $i \in I_{1}^{\prime}$. We estimate

$$
\sum_{j \in I_{1}^{\prime} \cup I_{2}^{\prime}, j \neq i} \ln \left|\cos 2 \pi a-\cos 2 \pi \theta_{j}\right|
$$

first.

Clearly,

$$
\begin{aligned}
& \sum_{j \in I_{1}^{\prime} \cup I_{2}^{\prime}, j \neq i} \ln \left|\cos 2 \pi a-\cos 2 \pi \theta_{j}\right| \\
& =\sum_{j \in I_{1}^{\prime} \cup I_{2}^{\prime}, j \neq i} \ln \left|\sin \pi\left(a+\theta_{j}\right)\right| \\
& \quad+\sum_{j \in I_{1}^{\prime} \cup I_{2}^{\prime}, j \neq i} \ln \left|\sin \pi\left(a-\theta_{j}\right)\right|+\left(2 q_{n}-1\right) \ln 2 \\
& =\Sigma_{+}+\Sigma_{-}+\left(2 q_{n}-1\right) \ln 2,
\end{aligned}
$$

where

$$
\Sigma_{+}=\sum_{j \in I_{1}^{\prime} \cup I_{2}^{\prime}, j \neq i} \ln |\sin \pi(a+\theta+j \alpha)|,
$$


and

$$
\Sigma_{-}=\sum_{j \in I_{1}^{\prime} \cup I_{2}^{\prime}, j \neq i} \ln |\sin \pi(a-\theta-j \alpha)| .
$$

Write $\Sigma_{+}$as the form

$$
\Sigma_{+}=\sum_{j \in I_{1}^{\prime}, j \neq i} \ln |\sin \pi(a+\theta+j \alpha)|+\sum_{j \in I_{2}^{\prime}} \ln |\sin \pi(a+\theta+j \alpha)| .
$$

We will estimate

$$
\sum_{j \in I_{1}^{\prime}, j \neq i} \ln |\sin \pi(a+\theta+j \alpha)| \text { and } \sum_{j \in I_{2}^{\prime}} \ln |\sin \pi(a+\theta+j \alpha)|,
$$

respectively.

On the one hand,

$$
\begin{aligned}
& \sum_{j \in I_{1}^{\prime}, j \neq i} \ln |\sin \pi(a+\theta+j \alpha)| \\
& =\sum_{j \in I_{1}^{\prime}} \ln |\sin \pi(a+\theta+j \alpha)|-\ln |\sin \pi(a+\theta+i \alpha)| \\
& =\sum_{k=1}^{q_{n}} \ln |\sin \pi(x+k \alpha)|-\ln |\sin \pi(a+\theta+i \alpha)| \\
& =\sum_{k=1, k \neq k_{0}}^{q_{n}} \ln |\sin \pi(x+k \alpha)|+\ln \left|\sin \pi\left(x+k_{0} \alpha\right)\right|-\ln |\sin \pi(a+\theta+i \alpha)|,
\end{aligned}
$$

where

$$
x=a+\theta-\left(\left[\frac{2}{3} q_{n}\right]+1\right) \alpha
$$

and $k_{0}$ satisfies

$$
\left|\sin \pi\left(x+k_{0} \alpha\right)\right|=\min _{1 \leq k \leq q_{n}}|\sin \pi(x+k \alpha)| .
$$

In Lemma 3.1, let $m_{k}=0, k=1,2, \ldots q_{n}$, by the second equality of (3.3), one has

$$
\sum_{k=1, k \neq k_{0}}^{q_{n}} \ln |\sin \pi(x+k \alpha)| \leq-\left(q_{n}-1\right) \ln 2+C \ln q_{n} .
$$


Since

$$
\ln \left|\sin \pi\left(x+k_{0} \alpha\right)\right| \leq \ln |\sin \pi(a+\theta+i \alpha)|
$$

(by the minimality of $k_{0}$ ), we have

$$
\sum_{j \in I_{1}^{\prime}, j \neq i} \ln |\sin \pi(a+\theta+j \alpha)| \leq-\left(q_{n}-1\right) \ln 2+C \ln q_{n} .
$$

On the other hand,

$$
\begin{aligned}
& \sum_{j \in I_{2}^{\prime}} \ln |\sin \pi(a+\theta+j)| \\
& \quad=\sum_{k=1}^{q_{n}} \ln \left|\sin \pi\left(x+\left(k+m_{k}\right) \alpha\right)\right| \\
& \quad=\sum_{k=1, k \neq k_{0}}^{q_{n}} \ln \left|\sin \pi\left(x+\left(k+m_{k}\right) \alpha\right)\right|+\ln \left|\sin \pi\left(x+\left(k_{0}+m_{k_{0}}\right) \alpha\right)\right|,
\end{aligned}
$$

where

$$
\begin{gathered}
x=a+\theta+\left(-\left[\frac{2}{3} q_{n}\right]+q_{n}-1\right) \alpha, \\
m_{k}=0, \quad \text { for } 1 \leq k \leq 2\left[\frac{2}{3} q_{n}\right]-q_{n}-1,
\end{gathered}
$$

and

$$
m_{k}=\ell-1, \quad \text { for } 2\left[\frac{2}{3} q_{n}\right]-q_{n} \leq k \leq q_{n},
$$

and $k_{0}$ satisfies

$$
\mid \sin \pi\left(x+\left(k_{0}+m_{k_{0}} \alpha\right)\left|=\min _{1 \leq k \leq q_{n}}\right| \sin \pi\left(x+\left(k+m_{k}\right) \alpha\right) \mid .\right.
$$

By the second equality of (3.3) again, one has

$$
\sum_{k=1, k \neq k_{0}}^{q_{n}} \ln \left|\sin \pi\left(x+\left(k+m_{k}\right) \alpha\right)\right| \leq-\left(q_{n}-1\right) \ln 2+C \ln q_{n} .
$$

In addition

$$
\ln \mid \sin \pi\left(x+\left(k_{0}+m_{k_{0}} \alpha\right) \mid \leq 0,\right.
$$

and one has

$$
\sum_{j \in I_{2}^{\prime}} \ln |\sin \pi(a+\theta+j \alpha)| \leq-\left(q_{n}-1\right) \ln 2+C \ln q_{n} .
$$


Putting (3.5), (3.6), and (3.7) together, we have

$$
\Sigma_{+} \leq-2 q_{n} \ln 2+C \ln q_{n} .
$$

We are now in the position to estimate $\Sigma_{-}$. In order to avoid repetition, we omit some details. Similarly, $\Sigma_{-}$consists of 2 terms of the form as (3.3), plus two terms of the form $\min _{k=1, \ldots, q_{n}} \ln \left|\sin \pi\left(x+\left(k+m_{k} q_{n}\right) \alpha\right)\right|$, where $m_{k} \in\{0,(\ell-1)\}$, $k=1, \ldots, q_{n}$, minus $\ln \left|\sin \pi\left(a-\theta_{i}\right)\right|$. Following the estimate of $\Sigma_{+}$,

$$
\Sigma_{-} \leq-2 q_{n} \ln 2+C \ln q_{n} .
$$

Putting (3.8) and (3.9) into (3.4), we obtain

$$
\sum_{j \in I_{1} \cup I_{2} j \neq i} \ln \left|\cos 2 \pi a-\cos 2 \pi \theta_{j}\right| \leq-2 q_{n} \ln 2+C \ln q_{n} .
$$

The estimate of

$$
\sum_{j \in I_{1}^{\prime} \cup I_{2}^{\prime}, j \neq i} \ln \left|\cos 2 \pi \theta_{i}-\cos 2 \pi \theta_{j}\right|
$$

require a bit more work.

It is easy to see that

$$
\sum_{j \in I_{1}^{\prime} \cup I_{2}^{\prime}, j \neq i} \ln \left|\cos 2 \pi \theta_{i}-\cos 2 \pi \theta_{j}\right|=\Sigma_{+}^{\prime}+\Sigma_{-}^{\prime}+\left(2 q_{n}-1\right) \ln 2,
$$

where

$$
\Sigma_{+}^{\prime}=\sum_{j \in I_{1} \cup I_{2}, j \neq i} \ln |\sin \pi(2 \theta+(i+j) \alpha)|
$$

and

$$
\Sigma_{-}^{\prime}=\sum_{j \in I_{1} \cup I_{2}, j \neq i} \ln |\sin \pi(i-j) \alpha| .
$$

Firstly, we estimate $\Sigma_{+}^{\prime}$. Similarly, $\Sigma_{+}^{\prime}$ consists of 2 terms of the form as (3.3), plus two terms of the form

$$
\min _{k=1, \ldots, q_{n}} \ln \left|\sin \pi\left(x+\left(k+m_{k} q_{n}\right) \alpha\right)\right|,
$$

where $m_{k} \in\{0,(\ell-1)\}, k=1, \ldots, q_{n}$, minus $\ln |\sin 2 \pi(\theta+i \alpha)|$. 
Following the above arguments and using the first inequality of (3.3), we obtain

$$
\Sigma_{+}^{\prime}>-2 q_{n} \ln 2-C \ln q_{n}+2 \min _{j, i \in I_{1} \cup I_{2}} \ln |\sin \pi(2 \theta+(j+i) \alpha)| .
$$

Thus it is enough to estimate the last term in (3.12). By the hypothesis $\theta \notin \mathcal{R}$, one has

$$
\min _{j, i \in\left[-2 q_{n}, 2 q_{n}-1\right]}|\sin \pi(2 \theta+(j+i) \alpha)|>\frac{1}{16 q_{n}^{2}} \text { for large } \mathrm{n} .
$$

If $k \in I_{2}$, let

$$
\ell_{k}=\ell-1 \quad \text { and } \quad k^{\prime}=k-\ell_{k} q_{n}
$$

if $k \in I_{1}$, let

$$
\ell_{k}=0 \quad \text { and } \quad k^{\prime}=k .
$$

Then $i^{\prime}, j^{\prime} \in\left[-2 q_{n}, 2 q_{n}-1\right]$. If $q_{n+1}>q_{n}^{100}$, it is easy to verify that

$$
\left|\ell_{k} \Delta_{n}\right|<\frac{1}{q_{n}^{5}}
$$

Combining with (3.13), we have, for any $i, j \in I_{1} \cup I_{2}$,

$$
\begin{aligned}
& |\sin \pi(2 \theta+(j+i) \alpha)| \\
& \quad=\mid \sin \pi\left(2 \theta+\left(j^{\prime}+i^{\prime}\right) \alpha\right) \cos \pi\left(\ell_{i}+\ell_{j}\right) \Delta_{n} \\
& \quad \quad \pm \cos \pi\left(2 \theta+\left(j^{\prime}+i^{\prime}\right) \alpha\right) \sin \pi\left(\ell_{i}+\ell_{j}\right) \Delta_{n} \mid \\
& \quad>\frac{1}{100 q_{n}^{2}}
\end{aligned}
$$

(the \pm depending on the sign of $q_{n} \alpha-p_{n}$ ).

If $q_{n+1} \leq q_{n}^{100}$, we also have

$$
|\sin \pi(2 \theta+(j+i) \alpha)|>\frac{1}{100 q_{n}^{200}}, \quad \text { for any } i, j \in I_{1} \cup I_{2},
$$

Thus, by (3.12), (3.14), and (3.15), one has

$$
\Sigma_{+}^{\prime}>-2 q_{n} \ln 2-C \ln q_{n} .
$$


Similarly, $\Sigma_{-}^{\prime}$ consists of 2 terms of the form as (3.3) plus the minimum term (because $\min _{j \in I_{1}^{\prime}}|\sin \pi(i-j) \alpha|=0$, then $\sum_{j \in I_{1}^{\prime}, j \neq i} \ln |\sin \pi(i-j) \alpha|$ is exactly of the form (3.3)). It follows that

$$
\Sigma_{-}^{\prime}>-2 q_{n} \ln 2-C \ln q_{n}+\min _{j \in I_{1} \cup I_{2}, j \neq i} \ln |\sin \pi((j-i) \alpha)| .
$$

We are now in the position to estimate the last term in (3.17). Note that for any $i \in I_{1} \cup I_{2}$, there is only one $\tilde{\imath} \in I_{1} \cup I_{2}$ such that $|i-\tilde{\imath}|=q_{n}$ or $\ell q_{n}$. It is easy to check

$$
\begin{aligned}
\ln |\sin \pi(i-\tilde{l}) \alpha| & \geq \min \left\{\ln \left|\sin \pi q_{n} \alpha\right|, \ln \left|\sin \pi \ell q_{n} \alpha\right|\right\} \\
& >-\ln q_{n+1}-C
\end{aligned}
$$

since

$$
\Delta_{n} \geq \frac{1}{2 q_{n+1}}
$$

If $j \neq i, \tilde{\imath}$ and $j \in I_{1} \cup I_{2}$, then

$$
j-i=r+m_{j}^{\prime} q_{n}, \quad \text { with } 1 \leq|r|<q_{n} \text { and }\left|m_{j}^{\prime}\right| \leq \ell .
$$

Thus by (3.1) and (3.2), one has

$$
\|r \alpha\|_{\mathbb{R} / \mathbb{Z}} \geq \Delta_{n-1} \geq \frac{1}{2 q_{n}}
$$

and

$$
\begin{aligned}
\min _{j \in I_{1} \cup I_{2} j \neq i, \tilde{l}} \ln |\sin \pi(j-i) \alpha| & >\ln \left(\|r \alpha\|_{\mathbb{R} / \mathbb{Z}}-\ell \Delta_{n}\right)-C \\
& >-\ln q_{n}-C,
\end{aligned}
$$

since

$$
\ell \Delta_{n}<\frac{1}{10 q_{n}}
$$

for $n$ large enough.

By (3.18) and (3.19), one has

$$
\min _{j \in I_{1} \cup I_{2} j \neq i} \ln |\sin \pi(j-i) \alpha|>-\ln q_{n+1}-C \ln q_{n} .
$$


By the definition of

$$
\beta=\limsup _{n \rightarrow \infty} \frac{\ln q_{n+1}}{q_{n}},
$$

equation (3.17) becomes

$$
\begin{aligned}
\Sigma_{-}^{\prime} & >-2 q_{n} \ln 2-\ln q_{n+1}-C \ln q_{n} \\
& >-2 q_{n} \ln 2-(\beta+\varepsilon) q_{n}-C \ln q_{n},
\end{aligned}
$$

for large $n$.

By (3.11), (3.16), and (3.20),

$$
\sum_{j \in I_{1}^{\prime} \cup I_{2}^{\prime}, j \neq i} \ln \left|\cos 2 \pi \theta_{i}-\cos 2 \pi \theta_{j}\right|>-2 q_{n} \ln 2-(\beta+\varepsilon) q_{n}-C \ln q_{n} .
$$

Together with (3.10), we obtain

$\sum_{j \in I_{1}^{\prime} \cup I_{2}^{\prime}, j \neq i} \ln \left|\cos 2 \pi a-\cos 2 \pi \theta_{j}\right|-\ln \left|\cos 2 \pi \theta_{i}-\cos 2 \pi \theta_{j}\right|<(\beta+\varepsilon) q_{n}+C \ln q_{n}$.

This implies

$$
\max _{x \in[-1,1]} \max _{i=1, \ldots, k+1} \prod_{j=1, j \neq i}^{k+1} \frac{\left|x-\cos 2 \pi \theta_{j}\right|}{\left|\cos 2 \pi \theta_{i}-\cos 2 \pi \theta_{j}\right|}<e^{\left(2 q_{n}-1\right)\left(\frac{\beta}{2}+\varepsilon\right)}
$$

for large enough $n$.

In Lemma 2.2, let $k=2 q_{n}-1, \epsilon_{1}=\frac{\beta}{2}+\eta$ and $\epsilon=\frac{\beta}{2}+C \eta$. Clearly, $\epsilon_{1}<\epsilon$. Thus there exists some $j_{0} \in I_{1} \cup I_{2}$ such that $\theta_{j_{0}} \notin A_{2 q_{n}-1, \ln \lambda-\frac{\beta}{2}-C \eta}$ for $n$ large enough.

STEP 2. We will show that $\theta_{j} \in A_{2 q_{n}-1, \ln \lambda-\frac{\beta}{2}-C \eta}$ for all $j \in I_{1}$.

Lemma 3.2. Suppose $k \in\left[-2 q_{n}, 2 q_{n}\right]$ and

$$
d=\operatorname{dist}\left(k,\left\{m q_{n}\right\}_{m \geq 0}\right) \geq \frac{q_{n}}{4},
$$

then, for sufficiently large $n$,

$$
|\phi(k)|<\exp (-(\ln \lambda-C \eta) d) .
$$


Proof. We will use block resolvent expansion to prove this lemma. By hypothesis $k \in\left[-2 q_{n}, 2 q_{n}\right]$, there exists some $m \in\{-2,-1,0,1\}$ such that

$$
m q_{n} \leq k<(m+1) q_{n} .
$$

For any $y \in\left[m q_{n}+\eta q_{n}+1,(m+1) q_{n}-\eta q_{n}-1\right]$, apply Theorem 2.2 with $\varepsilon=\eta$. Note that in case (i), we have

$$
\ln \lambda+9 \ln \left(s q_{n-1} / q_{n}\right) / q_{n-1}-\eta \geq \ln \lambda-C \eta,
$$

for large $n$. Thus $y$ is regular with $t=\ln \lambda-C \eta$. Moreover, there exists an interval

$$
I(y)=\left[x_{1}, x_{2}\right] \subset\left[(m-1) q_{n},(m+2) q_{n}\right]
$$

such that $y \in I(y)$ and

$$
\operatorname{dist}(y, \partial I(y)) \geq \frac{\eta}{2}|I(y)| \geq \frac{\eta}{2} q_{n-1}
$$

and

$$
\left|G_{I(y)}\left(y, x_{i}\right)\right|<e^{-(\ln \lambda-C \eta)\left|y-x_{i}\right|}, \quad i=1,2,
$$

where $\partial I(y)$ is the boundary of the interval $I(y)$, i.e., $\left\{x_{1}, x_{2}\right\}$, and recall that $|I(y)|$ is the number of $I(y)$, i.e.,

$$
|I(y)|=x_{2}-x_{1}+1
$$

For $z \in \partial I(y)$, let $z^{\prime}$ be the neighbor of $z$ (i.e., $\left.\left|z-z^{\prime}\right|=1\right)$ not belonging to $I(y)$. If

$$
x_{2}+1<(m+1) q_{n}-\eta q_{n} \text { or } \quad x_{1}-1>m q_{n}+\eta q_{n},
$$

then we can expand $\phi\left(x_{2}+1\right)$ or $\phi\left(x_{1}-1\right)$ as (2.4). We can continue this process until we arrive to $z$ such that

$$
z+1 \geq(m+1) q_{n}-\eta q_{n} \quad \text { or } \quad z-1 \leq m q_{n}+\eta q_{n},
$$

or the iterating number reaches

$$
\left[\frac{2 d}{\eta q_{n-1}}\right]
$$


Thus, by (2.4)

$$
\phi(k)=\sum_{s ; z_{i+1} \in \partial I\left(z_{i}^{\prime}\right)} G_{I(k)}\left(k, z_{1}\right) G_{I\left(z_{1}^{\prime}\right)}\left(z_{1}^{\prime}, z_{2}\right) \ldots G_{I\left(z_{s}^{\prime}\right)}\left(z_{s}^{\prime}, z_{s+1}\right) \phi\left(z_{s+1}^{\prime}\right),
$$

where in each term of the summation one has

$$
m q_{n}+\eta q_{n}+1<z_{i}<(m+1) q_{n}-\eta q_{n}-1, \quad i=1, \ldots, s,
$$

and either

$$
z_{s+1} \notin\left[m q_{n}+\eta q_{n}+2,(m+1) q_{n}-\eta q_{n}-2\right], \quad s+1<\left[\frac{2 d}{\eta q_{n-1}}\right],
$$

or

$$
s+1=\left[\frac{2 d}{\eta q_{n-1}}\right]
$$

When $(*)$, then, by (3.22),

$$
\begin{aligned}
& \left|G_{I(k)}\left(k, z_{1}\right) G_{I\left(z_{1}^{\prime}\right)}\left(z_{1}^{\prime}, z_{2}\right) \ldots G_{I\left(z_{s}^{\prime}\right)}\left(z_{s}^{\prime}, z_{s+1}\right) \phi\left(z_{s+1}^{\prime}\right)\right| \\
& \quad<e^{-(\ln \lambda-C \eta)\left(\left|k-z_{1}\right|+\sum_{i=1}^{S}\left|z_{i}^{\prime}-z_{i+1}\right|\right)} q_{n}^{C} \\
& \quad<e^{-(\ln \lambda-C \eta)\left(\left|k-z_{s+1}\right|-(s+1)\right)} q_{n}^{C} \\
& \quad<e^{-(\ln \lambda-C \eta)\left(d-\eta q_{n}-4-\frac{2 d}{\eta q_{n}-1}\right)} q_{n}^{C}
\end{aligned}
$$

since

$$
\left|\phi\left(z_{s+1}^{\prime}\right)\right| \leq\left(1+\left|z_{s+1}^{\prime}\right|\right)^{C} \leq q_{n}^{C} .
$$

When $(* *)$, then, using (3.21) and (3.22), we obtain

$$
\begin{aligned}
& \left|G_{I(k)}\left(k, z_{1}\right) G_{I\left(z_{1}^{\prime}\right)}\left(z_{1}^{\prime}, z_{2}\right) \ldots G_{I\left(z_{s}^{\prime}\right)}\left(z_{s}^{\prime}, z_{s+1}\right) \phi\left(z_{s+1}^{\prime}\right)\right| \\
& \quad<e^{-(\ln \lambda-C \eta) \frac{\eta q_{n-1}}{2}\left[\frac{2 d}{\eta q_{n-1}}\right]_{n}} q_{n}^{C}
\end{aligned}
$$

Finally, note that the total number of terms in (3.23) is at most $2^{\left[\frac{2 d}{n q_{n-1}}\right]}$ and $d \geq \frac{q_{n}}{4}$. Combining with (3.24) and (3.25), we obtain

$$
|\phi(k)|<e^{-(\ln \lambda-C \eta) d}
$$

for large $n$. 
Remark 3.2. Under the hypothesis of Lemma 3.2, Avila and Jitomirskaya only prove that

$$
|\phi(k)|<\exp \left(-(\ln \lambda-\varepsilon) \frac{d}{2}\right)
$$

We give the refined version.

Theorem 3.2. For any $b \in\left[-\frac{5}{3} q_{n},-\frac{1}{3} q_{n}\right]$, we have

$$
\theta+\left(b+q_{n}-1\right) \alpha \in A_{2 q_{n}-1,2 \ln \lambda / 3+C \eta}
$$

if $n$ is large enough, i.e., for all $j \in I_{1}, \theta_{j} \in A_{2 q_{n}-1,2 \ln \lambda / 3+C \eta}$.

Proof. Let

$$
b_{1}=b-1 \quad \text { and } \quad b_{2}=b+2 q_{n}-1 .
$$

Applying Lemma 3.2, one obtains that for $i=1,2$,

$$
\left|\phi\left(b_{i}\right)\right| \leq \begin{cases}e^{-(\ln \lambda-C \eta)\left(2 q_{n}+b\right)} & \text { if }-\frac{5 q_{n}}{3} \leq b \leq-\frac{3 q_{n}}{2}, \\ e^{-(\ln \lambda-C \eta)\left|q_{n}+b\right|} & \text { if }-\frac{3 q_{n}}{2}<b<-\frac{q_{n}}{2} \text { and }\left|b+q_{n}\right|>\frac{1}{4} q_{n}, \\ e^{(\ln \lambda-C \eta) b} & \text { if }-\frac{q_{n}}{2} \leq b \leq-\frac{q_{n}}{3} .\end{cases}
$$

In (2.4), let

$$
I=\left[b, b+2 q_{n}-2\right] \quad \text { and } \quad x=0 ;
$$

we get, for $n$ large enough,

$$
\begin{aligned}
& \max \left(\left|G_{I}(0, b)\right|,\left|G_{I}\left(0, b+2 q_{n}-2\right)\right|\right) \\
& \geq \begin{cases}e^{(\ln \lambda-C \eta)\left(2 q_{n}+b\right)} & \text { if }-\frac{5 q_{n}}{3} \leq b \leq-\frac{3 q_{n}}{2}, \\
e^{(\ln \lambda-C \eta)\left|q_{n}+b\right|} & \text { if }-\frac{3 q_{n}}{2}<b<-\frac{q_{n}}{2} \text { and }\left|b+q_{n}\right|>\frac{1}{4} q_{n} ; \\
e^{-(\ln \lambda-C \eta) b}, & \text { if }-\frac{q_{n}}{2} \leq b \leq-\frac{q_{n}}{3}, \\
e^{-C \eta}, & \text { if }\left|b+q_{n}\right| \leq \frac{1}{4} q_{n},\end{cases}
\end{aligned}
$$

since $\phi(0)=1$ and $|\phi(k)| \leq(1+|k|)^{C}$. 
Let

$$
\varepsilon=\eta
$$

in (2.3), and let

$$
I=\left[b, b+2 q_{n}-2\right], \quad y=0, \quad k=2 q_{n}-1
$$

in (2.1) and (2.2). After careful computation, we obtain

$$
\begin{aligned}
& \mid Q_{2 q_{n}-1}\left(\cos 2 \pi\left(\theta+\left(b+q_{n}-1\right) \alpha\right) \mid\right. \\
& \quad=\left|P_{2 q_{n}-1}(\theta+b \alpha)\right| \\
& \quad \leq \min \left\{\left|G_{I}(0, b)\right|^{-1} e^{(\ln \lambda+\eta)\left(b+2 q_{n}-2\right)},\left|G_{I}\left(0, b+2 q_{n}-2\right)\right|^{-1} e^{-(\ln \lambda+\eta) b}\right\} \\
& \quad \leq e^{\left(2 q_{n}-1\right)(2 \ln \lambda / 3+C \eta)} .
\end{aligned}
$$

Since $\ln \lambda>\frac{3 \beta}{2}$, thus

$$
\frac{2 \ln \lambda}{3}+C \eta<\ln \lambda-\frac{\beta}{2}-C \eta
$$

for small enough $\eta$. By STEP 1 and STEP 2, we have

$$
\theta_{j} \in A_{2 q_{n}-1, \ln \lambda-\frac{\beta}{2}-C \eta} \text { for all } j \in I_{1} .
$$

This implies there exists some $j_{0} \in I_{2}$ such that $\theta_{j_{0}} \notin A_{2 q_{n}-1, \ln \lambda-\frac{\beta}{2}-C \eta}$.

STEP 3. Establish the regularity for $y$.

Theorem 3.3. For some $t>0, y$ is $\left(t, 2 q_{n}-1\right)$-regular with $\delta=1 / 5$ for large enough $n$.

Proof. According to the previous two steps, there exists some

$$
\theta_{j_{0}} \notin A_{2 q_{n}-1, \ln \lambda-\frac{\beta}{2}-C \eta}, \quad \text { for } j_{0} \in I_{2} .
$$

Set

$$
I=\left[j_{0}-q_{n}+1, j_{0}+q_{n}-1\right]=\left[x_{1}, x_{2}\right] .
$$

In (2.3), let $\varepsilon=\eta$; combining with (2.1) and (2.2), it is easy to verify that

$$
\left|G_{I}\left(y, x_{i}\right)\right|<e^{(\ln \lambda+\eta)\left(2 q_{n}-2-\left|y-x_{i}\right|\right)-2 q_{n}\left(\ln \lambda-\frac{\beta}{2}-C \eta\right)} .
$$

By a simple computation $\left|y-x_{i}\right| \geq\left(\frac{2}{3}-C \eta\right) q_{n}$; then

$$
\left|G_{I}\left(y, x_{i}\right)\right|<e^{-\left|y-x_{i}\right|\left(\ln \lambda-\frac{3}{2} \beta-C \eta\right)},
$$

for large enough $n$. Select $\eta$ small enough such that $t=\ln \lambda-\frac{3 \beta}{2}-C \eta>0$, then $y$ is $\left(\ln \lambda-\frac{3 \beta}{2}-C \eta, 2 q_{n}-1\right)$-regular with $\delta=1 / 5$. 


\section{The proof of Theorem 2.1}

Now that the regularity for $y$ is established, we will use block resolvent expansion again to prove Theorem 2.1.

Proof of Theorem 2.1. Give some $k$ with $k>q_{n}$ and $n$ large enough. Using Theorems 2.2 and 3.3, for all $y \in\left[b_{n}, 2 k\right]$, then there exists an interval

$$
I(y)=\left[x_{1}, x_{2}\right] \subset[-4 k, 4 k], \quad \text { with } y \in I(y),
$$

such that

$$
\operatorname{dist}(y, \partial I(y))>\frac{\eta}{2} q_{n-1}
$$

and

$$
\left|G_{I(y)}\left(y, x_{i}\right)\right|<e^{-\left(\ln \lambda-\frac{3}{2} \beta-C \eta\right)\left|y-x_{i}\right|}, \quad i=1,2 .
$$

As in the proof of Lemma 3.2, denote by $\partial I(y)$ the boundary of the interval $I(y)$. For $z \in \partial I(y)$, let $z^{\prime}$ be the neighbor of $z$, (i.e., $\left|z-z^{\prime}\right|=1$ ) not belonging to $I(y)$.

If

$$
x_{2}+1<2 k \text { or } \quad x_{1}-1>b_{n},
$$

then we can expand $\phi\left(x_{2}+1\right)$ or $\phi\left(x_{1}-1\right)$ as (2.4). We can continue this process until we arrive to $z$ such that

$$
z+1 \geq 2 k \quad \text { or } \quad z-1 \leq b_{n},
$$

or the iterating number reaches

$$
\left[\frac{2 k}{\eta q_{n-1}}\right]
$$

By (2.4),

$$
\phi(k)=\sum_{s ; z_{i+1} \in \partial I\left(z_{i}^{\prime}\right)} G_{I(k)}\left(k, z_{1}\right) G_{I\left(z_{1}^{\prime}\right)}\left(z_{1}^{\prime}, z_{2}\right) \ldots G_{I\left(z_{s}^{\prime}\right)}\left(z_{s}^{\prime}, z_{s+1}\right) \phi\left(z_{s+1}^{\prime}\right),
$$

where in each term of the summation we have

$$
b_{n}+1<z_{i}<2 k-1, \quad i=1, \ldots, s,
$$

and either

$$
z_{s+1} \notin\left[b_{n}+2,2 k-2\right], \quad s+1<\left[\frac{2 k}{\eta q_{n-1}}\right],
$$

or

$$
s+1=\left[\frac{2 k}{\eta q_{n-1}}\right] .
$$


When ( $\star)$, then, by (4.2), one has

$$
\begin{aligned}
& \left|G_{I(k)}\left(k, z_{1}\right) G_{I\left(z_{1}^{\prime}\right)}\left(z_{1}^{\prime}, z_{2}\right) \ldots G_{I\left(z_{s}^{\prime}\right)}\left(z_{s}^{\prime}, z_{s+1}\right) \phi\left(z_{s+1}^{\prime}\right)\right| \\
& \leq e^{-\left(\ln \lambda-\frac{3}{2} \beta-C \eta\right)\left(\left|k-z_{1}\right|+\sum_{i=1}^{S}\left|z_{i}^{\prime}-z_{i+1}\right|\right)} k^{C} \\
& \leq e^{-\left(\ln \lambda-\frac{3}{2} \beta-C \eta\right)\left(\left|k-z_{s+1}\right|-(s+1)\right)} k^{C} \\
& \leq \max \left\{e^{-\left(\ln \lambda-\frac{3}{2} \beta-C \eta\right)\left(k-b_{n}-4-\frac{2 k}{n q_{n-1}}\right)} k^{C},\right. \\
& \left.e^{-\left(\ln \lambda-\frac{3}{2} \beta-C \eta\right)\left(2 k-k-4-\frac{2 k}{\eta q_{n}-1}\right)} k^{C}\right\} .
\end{aligned}
$$

When $(\star \star)$, then, using (4.1) and (4.2), we obtain

$$
\begin{aligned}
& \left|G_{I(k)}\left(k, z_{1}\right) G_{I\left(z_{1}^{\prime}\right)}\left(z_{1}^{\prime}, z_{2}\right) \ldots G_{I\left(z_{s}^{\prime}\right)}\left(z_{s}^{\prime}, z_{s+1}\right) \phi\left(z_{s+1}^{\prime}\right)\right| \\
& \quad \leq e^{-\left(\ln \lambda-\frac{3}{2} \beta-C \eta\right) \frac{\eta q_{n-1}}{2}\left[\frac{2 k}{\eta q_{n-1}}\right]_{k} C} .
\end{aligned}
$$

Finally, note that the total number of terms in (4.3) is at most $2^{\left[\frac{2 k}{n q_{n-1}}\right]}$. Combining with (4.4) and (4.5), we obtain

$$
|\phi(k)| \leq e^{-\left(\ln \lambda-\frac{3}{2} \beta-C \eta\right) k}
$$

for large enough $n$ (or equivalently large enough $k$ ).

For $k<0$, the proof is similar. Thus

$$
|\phi(k)| \leq e^{-\left(\ln \lambda-\frac{3}{2} \beta-C \eta\right)|k|} \quad \text { if }|k| \text { is large enough. }
$$

This ends the proof of Theorem 2.1.

Corollary 4.1. Suppose $\lambda>e^{\frac{3}{2} \beta}$ and $\theta \notin \mathcal{R}$. If a solution $\Psi_{E}(k)$ satisfies

$$
H_{\lambda, \alpha, \theta} \Psi_{E}=E \Psi_{E}, \quad \text { with } \Psi_{E}(k) \leq(1+|k|)^{C} \text { and } E \in \Sigma_{\lambda, \alpha},
$$

then

$$
\limsup _{|k| \rightarrow \infty} \frac{\ln \left(\Psi_{E}^{2}(k)+\Psi_{E}^{2}(k+1)\right)}{2|k|} \leq-\left(\ln \lambda-\frac{3}{2} \beta\right) .
$$

In particular, for $\beta(\alpha)=0$

$$
\lim _{|k| \rightarrow \infty} \frac{\ln \left(\Psi_{E}^{2}(k)+\Psi_{E}^{2}(k+1)\right)}{2|k|}=-\ln \lambda .
$$


Proof. If $\beta(\alpha)>0$, in fact (4.7) holds for any $\eta>0$, this implies

$$
\limsup _{|k| \rightarrow \infty} \frac{\ln \left(\Psi_{E}^{2}(k)+\Psi_{E}^{2}(k+1)\right)}{2|k|} \leq-\left(\ln \lambda-\frac{3}{2} \beta\right) \quad \text { if } \beta>0 .
$$

If $\beta(\alpha)=0$, following [1] or [2], $k$ is $(t, \ell(k))$-regular for large $|k|$, with

$$
t=\ln \lambda-\varepsilon .
$$

By the method of block resolvent expansion as above, we can obtain

$$
\left|\Psi_{E}(k)\right|<e^{-(\ln \lambda-\varepsilon)|k|} \quad \text { if } k \text { is large enough, }
$$

thus

$$
\limsup _{|k| \rightarrow \infty} \frac{\ln \left(\Psi_{E}^{2}(k)+\Psi_{E}^{2}(k+1)\right)}{2|k|} \leq-\ln \lambda .
$$

By (4.10) and (4.11), we obtain (4.8).

By Furman's uniquely ergodic theorem (Corollary 2 in [7]),

$$
\liminf _{|k| \rightarrow \infty} \frac{\ln \left(\Psi_{E}^{2}(k)+\Psi_{E}^{2}(k+1)\right)}{2|k|} \geq-\ln \lambda .
$$

The last two inequalities imply (4.9).

\section{References}

[1] A. Avila and S. Jitomirskaya, The Ten Martini Problem. Ann. of Math. (2) 170 (2009), no. 1, 303-342. MR 2521117 Zbl 1166.47031

[2] A. Avila and S. Jitomirskaya, Almost localization and almost reducibility. J. Eur. Math. Soc. (JEMS) 12 (2010), no. 1, 93-131. MR 2578605 Zbl 1185.47028

[3] A. Avila and R. Krikorian, Reducibility or nonuniform hyperbolicity for quasiperiodic Schrödinger cocycles. Ann. of Math. (2) 164 (2006), no. 3, 911-940.

MR 2259248 Zbl 1138.47033

[4] Yu. M. Berezanskiı̆, Expansions in eigenfunctions of selfadjoint operators. Akademijá Nauk Ukrainskoŭ SSSR. Institut Matematiki Izdat. "Naukova Dumka”, Kiev, 1965. In Russian. English transl by R. Bolstein, J. M. Danskin, J. Rovnyak, and L. Shulman. Translations of Mathematical Monographs, 17. American Mathematical Society, Providence, R.I., 1968. MR 0222719 (Russian) MR 0222718 (transl.) Zbl 0142.37203 (Russian) Zbl 0157.16601 (transl.)

[5] J. Bourgain, Estimates on Green's functions, localization and the quantum kicked rotor model. Ann. of Math. (2) 156 (2002), no. 1, 249-294. MR 1935847 Zbl 1213.82054 
[6] J. Bourgain, Green function estimates for lattice Schrödinger operators and applications. Annals of Mathematics Studies, 158. Princeton University Press, Princeton, N.J., 2005. MR 2100420 Zbl 1137.35001

[7] A. Furman, On the multiplicative ergodic theorem for uniquely ergodic systems. Ann. Inst. H. Poincaré Probab. Statist. 33 (1997), no. 6, 797-815. MR 1484541 Zbl 0892.60011

[8] A. Ya. Gordon, S. Jitomirskaya, Y. Last, and B. Simon, Duality and singular continuous spectrum in the almost Mathieu equation. Acta Math. 178 (1997), 169-183. MR 1459260 Zbl 0897.34074

[9] P. G. Harper, Single band motion of conduction electrons in a uniform magnetic field. Proc. Phys. Soc. London A 68 (1955), 874-892. Zbl 0065.23708

[10] S. Jitomirskaya, Almost everything about the almost Mathieu operator. II. In D. Iagolnitzer (ed.), $\mathrm{XI}^{\text {th }}$ International Congress of Mathematical Physics. Proceedings of the congress held in Paris, July 18-23 and the ICMP Satellite Conference on Topology, Strings and Integrable Models held in Paris, July 25-28, 1994. International Press, Cambridge, MA, 1995, 373-382. MR 1370694 MR 1370662 (collection) Zbl 1052.82539 Zbl 0914.00104 (collection)

[11] S. Jitomirskaya, Metal-insulator transition for the almost Mathieu operator. Ann. of Math. (2) 150 (1999), no. 3, 1159-1175. MR 1740982 Zbl 0946.47018

[12] S. Jitomirskaya and B. Simon, Operators with singular continuous spectrum. III. Almost periodic Schrödinger operators. Comm. Math. Phys. 165 (1994), no. 1, 201-205. MR 1298948 Zbl 0830.34074

[13] Y. Last, Spectral theory of Sturm-Liouville operators on infinite intervals: a review of recent developments. In W. O. Amrein, A. M. Hinz, and D. B. Pearson (eds.), Sturm-Liouville theory. Past and present. Including papers from the International Colloquium held at the University of Geneva, Geneva, September 15-19, 2003, o commemorate the $200^{\text {th }}$ anniversary of the birth of Ch. F. Sturm. Birkhäuser, Basel, 2005, 99-120. MR 2145079 MR 2132131 (collection) Zbl 1098.39011 Zbl 1064.34001 (collection)

[14] B. Simon, Schrödinger operators in the twenty-first century. In A. Fokas, A. Grigoryan, T. Kibble, and B. Zegarlinski (eds.) Mathematical physics 2000. International congress, London, GB, 2000. Imperial College Press, London, 2000, 283-288. MR 1773049 MR 1773035 (collection) Zbl 1074.81521 Zbl 0949.00037 (collection)

[15] J. You and Q. Zhou, Embedding of analytic quasi-periodic cocycles into analytic quasi-periodic linear systems and its applications. Comm. Math. Phys. 323 (2013), no. 3, 975-1005. MR 3106500 Zbl 1286.37004 
Received October 1, 2013; revised October 21, 2014

Wencai Liu, School of Mathematical Sciences, Fudan University, Shanghai 200433, People's Republic of China

e-mail: liuwencai1226@gmail.com

Xiaoping Yuan, School of Mathematical Sciences, Fudan University, Shanghai 200433, People's Republic of China

e-mail: xpyuan@fudan.edu.cn 